\title{
1 Non-target impacts of fungicide disturbance on phyllosphere yeasts in different crop species
}

2 and management systems

3

4 Zachary A. Noel ${ }^{1,4, *}$, Reid Longley ${ }^{2, *}$, Gian Maria Niccolò Benucci ${ }^{1}$, Frances Trail ${ }^{1,3}$, Martin I.

5 Chilvers ${ }^{1}$, Gregory Bonito ${ }^{1,2, \#}$

$7 \quad{ }^{1}$ Department of Plant, Soil and Microbial Sciences. Michigan State University. East Lansing, MI 8 48824, U.S.A.

$9{ }^{2}$ Department of Microbiology and Molecular Genetics, Michigan State University, East Lansing, MI 10 48824, U.S.A.

$11{ }^{3}$ Department of Plant Biology. Michigan State University. East Lansing, MI 48824, U.S.A

$12{ }^{4}$ Current address: Department of Entomology and Plant Pathology, Auburn University, Auburn, AL 13 36849, U.S.A.

$39 *$ These authors contributed equally to this work

40 \# Corresponding author: bonito@msu.edu 


\section{Summary}

- Fungicides reduce fungal pathogen populations and are essential to food security. Fungicide disturbance of plant microbiomes has received limited attention. Understanding the impacts of fungicides on crop microbiomes in different cropping systems is vital to minimizing unintended consequences while maintaining their use for plant protection.

- We used amplicon sequencing of fungi and prokaryotes in maize and soybean microbiomes before and after foliar fungicide application in leaves and roots from plots under long-term no-till and conventional tillage managements. We examine fungicide disturbance and microbiome resilience across these treatments.

- Foliar fungicides directly affected phyllosphere fungal communities, but not root fungal communities or prokaryote communities. Impacts on fungal phyllosphere composition and resiliency were management-dependent and lasted more than thirty days. Fungicides lowered pathogen abundance in maize and soybean and decreased the abundance of Tremellomycetes yeasts, especially the Bulleribacidiaceae, including core microbiome members.

- Fungicide application reduced network complexity in the soybean phyllosphere. Bulleribacidiaceae often co-occurred with Sphingomonas and Hymenobacter in control plots, but co-occurrences were altered in fungicide plots. Results indicate that foliar fungicides lower pathogen and non-target fungal abundance and may impact prokaryotes indirectly. Notill management was more resilient following fungicide disturbance and recovery.

Keywords: Phyllosphere, fungicide, yeast, microbiome, disturbance, no-till

\section{Introduction}

Disturbances from chemical applications in agriculture reduce the abundance of pests and pathogens and are common in modern agricultural ecosystems (Rykiel, 1985; Glasby \& Underwood, 1996; Sullivan \& Sullivan, 2003; Landers et al., 2012; Shade et al., 2012). However, applying disturbance concepts to microbial communities can be challenging to assess recovery and analyze the full impacts. A lack of data on the impacts crop management combined with fungicide disturbances on the plant microbiome hinders developing novel strategies to minimize diversity loss, understand unintended consequences of these applications, and improve crop microbiomes' resilience. Observing fluctuations in taxa abundance and secondary effects mediated through microbial interactions 
following fungicide application opens the possibility for novel ecologically motivated strategies that promote microbiome stability or resilience following a fungicide application.

Fungicide use has become common in conventional agricultural systems. Yet, concerns remain about direct and indirect effects on non-targeted organisms, consequences (i.e., resistance), and negative impacts on the environment or human health (Hahn, 2014; Schaeffer et al., 2017b; Zubrod et al., 2019). The rapid evolution of fungicide resistance in plant and human pathogenic fungal populations can cause devastating epidemics in agricultural ecosystems, with spill-over effects to public health (Verweij et al., 2009; Delmas et al., 2017; Riat et al., 2018; McDonald et al., 2019). For example, there is substantial concern about the overuse of azole fungicides that have been linked to the resistance of Aspergillus fumigatus to antifungals in human infections (Riat et al. 2018; Verweij et al. 2009). Despite concerns, foliar fungicide applications in maize (Zea mays L.) and soybean (Glycine max L. Merr) are often made without substantial pathogen pressure due to perceived or marketed yield benefits (Wise \& Mueller, 2011; Kandel et al. 2021). A meta-analysis of soybeans demonstrated that foliar fungicide application in the absence of disease increased yield by $2.7 \%$. Still, economic analyses indicated that fungicides are less profitable without disease pressure (Kandel et al., 2021). While fungicides are necessary for crop protection, minimizing non-target effects and unintended consequences is critical in evaluating the sustainability of agricultural production systems.

Studies reporting fungicidal and pesticidal impacts to soil microbiomes (Imfeld \& Vuilleumier, 2012; Fournier et al., 2020) and aquatic organisms (Zubrod et al., 2019) are more abundant than those where fungicides are applied directly to the foliage, which is surprising considering the numerous phyllosphere pathogens and microorganisms present in this habitat. From the few studies focused on the plant phyllosphere, a consistent non-target effect is detected against phyllosphere yeasts. For example, one study on grapevine microbiomes reported minimal and transient impacts to the phyllosphere microbiome, including phyllosphere yeast abundance (Perazzolli et al., 2014). Similarly, repeated application of broad-spectrum fungicides has been shown through culture-based and culture-independent methods to decrease phyllosphere yeast richness (Dickinson \& Wallace, 1976; Southwell et al., 1999; Sapkota et al., 2015; Knorr et al., 2019).

Yeasts that inhabit the phyllosphere are well suited to oligotrophic and dynamic environmental conditions present on leaf surfaces and have been consequently used as biocontrol of plant pathogens (Freimoser et al., 2019). They are known to produce extracellular polysaccharides 
103 and surfactants, which may be necessary for creating or maintaining biofilms (Fonseca \& Inácio,

104 2006). Additionally, some phyllosphere yeasts, including species of basidiomycete yeasts in

105 Cryptococcus and Sporidiobolus, produce carotenoid compounds, which have antioxidant properties

106 and may protect the yeasts and potentially other resident microbes from stress in the phyllosphere

107 (Cobban et al., 2016). Phyllosphere yeast communities have also been linked to pollinator insects by

108 altering floral nectary chemistry, and fungicides can modify this relationship (Cadez et al., 2010;

109 Schaeffer et al., 2017a). However, no study has addressed the links between phyllosphere yeasts and

110 other phyllosphere residing microorganisms. While indirect and collective effects of removing single

111 species or groups of species from ecosystems proposed in ecological theory since the 1940s and have

112 been studied in various macro-organism contexts such as conservation biology, disturbance ecology,

113 and food web ecology, such effects are comparatively understudied in microbiomes (Tilman, 1999;

114 Ripple \& Beschta, 2005; Sahasrabudhe \& Motter, 2011). In microbiomes, network complexity (i.e.,

115 linkage density) has been correlated to ecosystem functioning and stability (Zhou et al., 2010; Wagg

116 et al., 2019). Consequently, indirect effects may be revealed through co-occurrence patterns, which

117 may not otherwise be seen through culturing or sequencing one target group.

118 Since the US Dust Bowl of the 1930s, soil conservation efforts have led to the steady adoption

119 of minimum or no-till agriculture management systems (Claassen et al., 2018). Cropping

120 management systems have been demonstrated to impact phyllosphere microbiomes (Gdanetz \& Trail,

121 2017; Longley et al., 2020). Crop management's effect on the resilience of foliar fungal communities

122 following fungicides has not been explored, but differing impacts of fungicides in different

123 agricultural managements are probable. In the only study of this kind, agricultural management

124 altered the response of microbial communities to tetraconazole fungicide application (Sułowicz et al.,

125 2016). However, the two management treatments existed in different locations confounding efforts to

126 distinguish fungicide management response effects from those of location (Karlsson et al., 2014).

127 Long-term experiments such as the Main Cropping Systems Experiment at the Kellogg Biological

128 Research Station, circumvent these confounding effects by applying all treatments at a single

129 location.

130 Here, we characterize non-target effects of foliar fungicides and resilience of the maize and

131 soybean leaf and root microbiomes in no-till and conventional plots of the Long-term Ecological

132 Research (LTER) Main Cropping Systems Experiment at the Kellogg Biological Station (KBS). Our

133 research objectives were three-fold: (1) to determine whether fungicides alter microbial diversity 
134 across plant compartments (e.g., leaves or roots), crop species (e.g., maize or soybean), or

135 management (conventional vs. no-till); (2) to identify non-target and indirect effects of fungicide

136 applications, and (3) determine if crop management alters the resiliency of the microbiome. We

137 hypothesized that fungicides would alter both maize and soybean microbial (fungal and prokaryotic)

138 diversity and network complexity. We predicted that this effect would be pronounced in the leaves

139 compared to roots. Additionally, given that plant microbiomes have been shown to differ under the

140 two management systems (Longley et al., 2020), we hypothesize that the response and recovery of

141 plant microbiomes following fungicides would also differ between the two management treatments.

142 This LTER site allows for a novel approach by eliminating any differences caused by location bias

143 and assessing the effect of fungicide application under long-term agricultural management. We apply

144 a novel microbiome network analysis approach to determine the impact fungicides have on

145 prokaryote-fungal co-occurrences in the plant microbiome. Finally, we used random forest models to

146 predict prokaryote taxa responsive to altered fungal diversity demonstrating the possible indirect

147 effects of fungicides.

148

149 Materials and Methods

150

\section{Experimental design}

152 Sample Site and Management Systems. All samples were collected from the no-till and

153 conventional management systems of maize (Zea mays L.), soybean (Glycine max L. Merr), and

154 wheat (Triticum aestivum L.) rotation of the main cropping experiment at Michigan State

155 University’s Kellogg Biological Station (KBS) Long Term Ecological Research (LTER) site. The site

156 contains one-hectare plots, consistently managed since 1989 (Robertson \& Hamilton, 2015). Each

157 agricultural management at the site is represented in six replicate plots spread randomly throughout to

158 avoid location bias. Fungicide micro-plots (10 feet wide x 20 feet long) were established within four

159 of the six replicate plots under no-till and conventional management treatments. Control samples

160 were taken from micro-plots of the same size directly next to the fungicide micro-plots. Samples were

161 taken from the middle of plots or towards the edge of the microplot that was not along the fungicide-

162 control (i.e., at least 6 meters apart) border to minimize the effect of spray drift. In 2017, the

163 fungicide Headline ${ }^{\circledR}$ was applied to maize foliage at a recommended rate of $877 \mathrm{ml} \mathrm{ha}^{-1}(12 \mathrm{fl} \mathrm{oz}$

164

acre $\left.^{-1}\right)$. Headline ${ }^{\circledR}$ contains the active ingredient pyraclostrobin, which acts as a mitochondrial 
165 respiration inhibitor. Pyraclostrobin is a local penetrant fungicide with translaminar movement and is 166 not translocated in the xylem (Latin, 2017). In 2018, soybean foliage was sprayed with Delaro ${ }^{\circledR}$

167 fungicide on 3 August 2018 (Bayer, Raleigh, NC, USA) at a recommended rate of $731 \mathrm{ml} \mathrm{ha}^{-1}$ (11 fl

$\left.168 \mathrm{oz} \mathrm{acre}^{-1}\right)$. The active ingredients in Delaro ${ }^{\circledR}$ are a combination of trifloxystrobin which inhibits 169 mitochondrial respiration, and prothioconazole, which inhibits ergosterol synthesis. Trifloxystrobin is 170 a local penetrant fungicide with translaminar movement and is not translocated in the xylem (Latin, 171 2017). Prothioconazole has acropetal penetrant activity and has weak basipetal movement (Augusto $172 \&$ Brenneman, 2012).

173

174 Sample collection and DNA extraction. In the summer of 2017, maize leaf and root samples were 175 collected at three time points throughout the growing season. The first sampling occurred before the 176 fungicide application on 26 June 2017 (V6 growth stage), the second was 9-days post fungicide 177 application (V8 growth stage), and the final sampling was 35-days post fungicide application (V15 178 growth stage). Leaves and roots from three plants from four replicate control or adjacent fungicide 179 treated plots of each no-till, and conventional management was sampled at each time point. Maize 180 leaves were sampled by removing two whole leaves from each plant and placing them into a sterile 181 Whirl Pak (Nasco, Madison, WI, USA) for transport back to the lab where they were stored at $-80^{\circ} \mathrm{C}$ 182 until they were lyophilized. At the V6 and V8 growth stage, the sixth and seventh leaf was sampled.

183 However, at the V15 growth stage, three leaves above the ear leaf were sampled. Roots were sampled 184 by removing whole plants from the soil and the entire root system to the soil line. Then roots were 185 washed in the field before being transported back to the lab, where roots were washed again with $1860.1 \%$ tween 20 (ThermoFisher Scientific, USA) and deionized water. Samples were stored at $-80^{\circ} \mathrm{C}$ 187 before being lyophilized for DNA extraction. Following lyophilization, the fine roots were removed 188 from the root system and used for DNA extraction.

189 In 2018, soybean leaves were sampled at three time points during the growing season, with 190 the first occurred before fungicide spray on 3 August 2018 (R3 growth stage), the second occurred 191 13-days post fungicide application (R4 growth stage), and the final occurring 33-days post fungicide 192 application (R6 growth stage) (Fehr et al., 1971). Soybean leaves were sampled with a flamed metal 193 hole punch, washed in 80\% ethanol, and flame sterilized between samples. Three 6-mm leaf discs 194 from three separate leaves were punched directly into an Eppendorf tube (Eppendorf, Germany) 195 containing $500 \mu \mathrm{l}$ of CSPL buffer (Omega Bio-Tek, Norcross, GA, USA). As with the maize roots, 
196 soybean roots were removed at the soil line and placed into a new Whirl-Pak (Nasco, Madison, WI,

197 USA) bag containing approximately $50 \mathrm{ml}$ of $0.1 \%$ tween 20 to remove the remaining soil. Root

198 samples were transported back to the lab, where roots were washed again with DI water, and samples

199 were stored at $-80^{\circ} \mathrm{C}$ until processing. Maize and soybean leaf and root tissue were pulverized for 2-

$200 \mathrm{~min}$ at a speed of $30 \mathrm{~Hz}$ with two 4-mm stainless balls in a TissueLyser II (Qiagen, Venlo,

201 Netherlands). Total DNA was extracted from plant tissues with the OMEGA Mag-Bind Plant DNA

202 Plus kit (Omega Bio-Tek, Norcross, GA, USA) following the manufacturer's instructions with the aid 203 of a KingFisher Flex ${ }^{\mathrm{TM}}$ liquid handling machine (ThermoFisher Scientific, USA). Five or six internal 204 negative extraction controls were included per 96-well plate in each DNA extraction.

205

206 Amplicon library preparation for ITS and 16s community profiling. Amplicon libraries were 207 prepared from a three-step PCR protocol as modified from Lundberg et al. (2013) and described by 208 Longley et al. (2020). In brief, fungal libraries were constructed around the ITS and were amplified 209 using the primers ITS1F and ITS4 (Gardes \& Bruns, 1993). Prokaryote libraries targeted the V4 210 region of 16S rRNA with the primers 515F and 806R (Caporaso et al., 2011). Supporting information 211 Tables S1, S2 and S3 describe PCR protocols, primers, and cycling conditions in detail. Amplicons 212 were purified with the SequalPrepTM Normalization Plate Kit (ThermoFisher Scientific, USA) and 213 then pooled and concentrated with Amicon ${ }^{\circledR}$ Ultra $0.5 \mathrm{~mL}$ filters (EMDmillipore, Germany).

214 Subsequently, the library was purified, and size selected with Agencourt AMPure XP magnetic beads 215 (Beckman Coulter, USA). Amplicon libraries were quantified and checked on the Agilent 4200 216 TapeStation DNA10000 and Kapa Illumina Library Quantification qPCR assays. All amplicon 217 libraries were then paired-end sequenced (300 bp reads) on an Illumina MiSeq with a v3 600 cycles 218 kit (Illumina, USA).

220 Bioinformatic sequence processing. Fungal ITS1 or prokaryotic 16S V4 reads were demultiplexed 221 in QIIME 1.9.1 (Caporaso et al., 2010). Forward and reverse prokaryote reads were merged using 222 QIIME 1.9.1. Only forward fungal ITS1 reads were used since reverse reads did not overlap. After 223 removing primers with Cutadapt 1.8.1 (Martin, 2011), fungal reads were trimmed to remove the conserved SSU and 28S regions. Reads were then quality filtered at an expected error threshold of 0.1 and truncated to equal length (fungi $200 \mathrm{bp}$; prokaryote $300 \mathrm{bp}$ ) in USEARCH 11.0.667 (Edgar, 
227 similarity using the UPARSE algorithm (Edgar, 2013). Using CONSTAX2 (Gdanetz et al., 2017;

228 Liber et al., 2021), the taxonomic classification of fungal and prokaryotic OTU's representative 229 sequences was performed against the UNITE eukaryote database, ver. 8.2 of 04.02.2020 (Abarenkov 230 et al., 2020) and SILVA, version 138 (Quast et al., 2013), respectively. To filter out non-target taxa 231 and OTUs unidentified at the Kingdom level, CONSTAX was run twice under different cutoff levels, 232 as suggested by Bowsher et al. (2020). Non-target taxa, OTUs not assigned to a Kingdom, and OTUs 233 identified as either chloroplast or mitochondria in either database were removed from further analysis 234 (Zhang et al., 2019).

236 Statistical analysis. Data were imported into R 4.0.3 (R core team 2020), and the R packages 237 phyloseq 1.24.2 (McMurdie \& Holmes, 2013) and vegan 2.5.3 (Jari Oksanen et al., 2018) were used 238 for most analyses. Samples with low sequencing coverage (less than 1000 reads) were removed from 239 the analysis. Contaminant OTUs (i.e., those prevalent in negative extraction controls) were removed 240 with the R package decontam (Davis et al., 2018). Before normalization, richness was assessed for 241 Prokaryotes and Fungi in the leaves and roots of each crop using the 'estimate_richness' function in 242 the phyloseq package. Results of alpha diversity analyses were plotted using the 'ggplot2' package 243 (Wickham, 2009). Then, sample read counts were normalized using the cumulative sum scaling 244 technique within the metagenomeSeq R package (Paulson et al., 2013). Differences in fungal and 245 prokaryotic community centroids were tested through permutational multivariate analysis of variance 246 (PERMANOVA) with the 'adonis2' function. Variation in multivariate dispersion was tested with the 247 'betadisper' function. More specific hypotheses were tested based on constrained analysis of principal 248 coordinates (CAP) (Anderson \& Willis, 2003).

Differentially abundant taxa resulting from fungicide application were identified by comparing fungicide treated plots to control plots through an analysis of the composition of microbiomes (ANCOM v 2.1) (Mandal et al., 2015). For differential abundance analysis, fungal OTUs (fOTU, hereafter) with a mean relative abundance less than $1.0^{-5}$ and fOTUs with zeros present in $95 \%$ samples were discarded from the analysis to avoid detecting fOTUs as significantly different based on stochasticity. Additionally, fOTUs that were never present in fungicide treated plots were not included. Fungal OTUs were determined to be significant if the $\mathrm{W}$ value was greater than $70 \%$ of the taxa tested based on Wilcoxon ranked sum test between additive log-ratio transformed data and a 
transient effects) were defined as fOTUs that were significantly less abundant following fungicide treatment but were not significantly less abundant from non-disturbed plots, after 33- or 34-dpf, for soybean or maize, respectively. Non-recovered taxa were defined as those fOTUs with significantly altered abundance following fungicide application and remained significantly altered for the remainder of the sampling. Additionally, a portion of non-recovered taxa was also locally extinct, which were defined as taxa present before fungicide application but having zero relative abundance following fungicide application in fungicide treated plots through the remainder of the sampling while being present in the control plots. Finally, taxa that did not have significantly altered abundance following fungicide application but then had significantly different abundance at a later sampling point (i.e., 33- or 34-dpf) were defined as indirect effects.

The core phyllosphere fungal and prokaryotic taxa from the non-fungicide disturbed no-till or conventional plots were identified based on each abundance and occupancy across space and time. Taxa that contributed to the last 2\% increase in Bray-Curtis distances were defined as the core (Shade \& Stopnisek, 2019).

We built random forest regression models to test the effect of altered prokaryote abundance through fungal diversity by using prokaryote abundances to predict fungal diversity. Random forest models were generated with the 'randomForest' function in the randomForest $\mathrm{R}$ package (Liaw \& Wiener, 2002). To remove redundant features and avoid overfitting models, we removed redundant OTUs with the 'Boruta' function in the package Boruta (Kursa \& Rudnicki, 2010). The method performs a top-down search for relevant OTUs by comparing the importance of the original OTUs from those selected at random. Models were tuned to achieve the lowest stable out-of-bag (OOB) error estimate possible, and the best mtry value (number of OTUs sampled at random in the entire pool for each tree at each split) was selected using the 'tuneRF' function in randomForest $\mathrm{R}$ package.

Network analysis was conducted on soybean and maize leaf samples to estimate cooccurrences among prokaryotic and fungal OTUs in each host and determine whether fungicides altered fungal-prokaryotic co-occurrences and network complexity (i.e., linkage density). For network analyses, soybean and maize fungal and prokaryotic OTU tables were filtered to exclude taxa with mean relative abundance below $1.0^{-5}$. A co-occurrence meta-matrix was estimated using the Meinshausen and Bühlmann algorithm within the SpiecEasi R package with the 'nlambda' set to 100 and with 'lambda.min.ratio' set to $1.0^{-2}$ (Kurtz et al., 2015). From this meta-matrix, subnetworks were created from taxa present within each sample. Then, network complexity was calculated for each 
subnetwork. The contribution of the Bulleribacidiaceae to network complexity was assessed by examining the change in the cumulative edge weights across subnetworks with prokaryotic genera.

\section{Results}

293 General sequencing results. The final fungal OTU table contained 20,844,912 ITS1 reads across 294554 samples, including 5315 fOTUs after filtering 36 contaminant fOTUs detected in negative controls. The median read depth was 30,370 ITS1 reads per sample. Prokaryotes contributed $29,691,681$ total reads across 555 samples with a median read depth of 47,590 reads per sample. A total of 14,291 prokaryote OTUs (pOTU, hereafter) were defined after filtering 55 contaminant pOTUs detected in the negative controls. Rarefaction curves verified that the median read depth adequately sampled the diversity present (Fig. S1).

Fungicides alter the maize and soybean leaf fungal richness. Following fungicide application, the richness of maize and soybean leaf fungal communities was significantly reduced $(\mathrm{P}<0.05)$ compared to that of non-sprayed control plots (Fig. S2). This reduction in fungal richness was observed in both crops in each management except no-till maize samples. This effect was most pronounced for the fungal classes of Dothideomycetes (target) and Tremellomycetes (non-target). However, in other fungal classes such as the Sordariomycetes, there was no significant difference in richness between control and fungicide treated samples following fungicide applications. Therefore, reductions in fungal richness were likely confined to specific fungal classes instead of being across all classes. Among prokaryotes, there were no consistent differences between control and fungicide samples in either crop. Likewise, there was no evidence that fungicides altered fungal or prokaryotic richness in roots.

\section{Fungicides alter the maize and soybean leaf fungal community composition. Fungal and}

314 prokaryote community composition varied significantly through time (i.e., days post fungicide or dpf) 315 and crop management in maize and soybean leaves and roots (Table S4; Table S5). Notably, before 316 fungicides were sprayed, crop management was shown to have a significant effect on the maize and 317 soybean phyllosphere fungal and prokaryotic communities (maize leaf fungi $\mathrm{R}^{2}=0.050, P=0.001$; 318 maize leaf prokaryotes $\mathrm{R}^{2}=0.038, P=0.005$; soybean leaf fungi $\mathrm{R}^{2}=0.058, P=0.020$; soybean leaf 319 prokaryotes $\mathrm{R}^{2}=0.046, P=0.049$ ). Furthermore, fungal and prokaryotic phyllosphere community 
compositions in control and treatment plots were indistinguishable from each other prior to applying

321 fungicide treatments (maize leaf fungi $\mathrm{R}^{2}=0.032, P=0.051$; maize leaf prokaryotes $\mathrm{R}^{2}=0.023, P=$

3220.418 ; soybean leaf fungi $\mathrm{R}^{2}=0.018, \mathrm{P}=0.483$; soybean leaf prokaryotes $\left.\mathrm{R}^{2}=0.035, P=0.128\right)$.

323 Despite this, changes to the fungal phyllosphere composition by fungicide treatments differed

324 depending on management (fungicide-management interaction) only in soybean, and not in maize the 325 phyllosphere (maize leaf fungi 9-dpf $\mathrm{R}^{2}=0.012, P=0.916$; soybean leaf fungi 13-dpf $\mathrm{R}^{2}=0.041, P$ $326=0.017$; soybean leaf fungi 33-dpf $\left.\mathrm{R}^{2}=0.039, P=0.015\right)$. There was no substantial evidence that

327 fungicides altered the composition of phyllosphere prokaryote communities, prokaryote root communities, or fungal root communities. Therefore, the variance explained due to the fungicide disturbance was examined for fungal phyllosphere communities before and after fungicide exposure while partitioning out the variation due to crop management by a constrained analysis of principal coordinates (CAP) (Fig. 1).

For soybean and maize leaves, no significant differences were observed prior to fungicide application $(P=0.51)$, but fungicide treatment had a significant effect on fungal leaf composition after fungicides were applied (13-dpf $13 \%$ variation $P<0.001$; 33-dpf $11 \%$ variation $P<0.001$ ) (Fig.1a-c). Similarly, the effect of fungicide disturbance on maize leaf fungal composition was not observed before fungicides were applied $(P=0.075)($ Fig. 1d). However, unlike soybean, there was no evidence the fungicide altered fungal composition longer than nine days $(9-\operatorname{dpf} 8 \%$ variation $P<$ $0.001 ; 34-d p f 3 \%$ variation $P=0.078$ ) (Fig. 1e, 1f). The non-significant beta dispersion tests across managements at 9-dpf or 34-dpf for maize (9-dpf conventional $P=0.369$, no-till $P=0.631 ; 34$-dpf conventional $P=0.364$, no-till $P=0.662)$ or 13 - and 33-dpf (13-dpf conventional $P=0.742$, conventional $P=0.866 ; 33$-dpf conventional $P=0.335$, no-till $P=0.123$ ) for soybean, indicate that

342 the effects of fungicide on fungal leaf composition are likely due to true differences in community composition rather than group dispersions (Table S6).

Fungicidal effects on network properties depend on crop management. In soybean under conventional and no-till management, network complexity was not significantly different before fungicide application (conventional $P=0.13$; no-till $P=0.93$ ), but was significantly lower than control plots 13-dpf (conventional $P=0.0015$; no-till $P=0.3$ ) (Fig. 2a). However, after one month, the soybean no-till network complexity had recovered $(P=0.12)$, whereas the conventional treatment 
351 in network complexity can partially be explained by a reduction in the number of nodes (i.e., OTUs)

352 since the average number of nodes per network also followed this same trend (Fig. 2b). These same

353 effects were not observed in maize. Fungicide disturbance was followed by the loss of network

354 complexity mainly through node loss, but crops and crop management impacted network properties

355 under fungicide disturbance. To investigate these trends more closely, we investigated the specific

356 fungal taxa affected through differential abundance analysis.

358 Identification of fungicide-affected fOTUs. To determine which fungal taxa were significantly 359 affected by fungicide application, a differential abundance analysis was conducted with ANCOM 360 (Table S7). In total, the abundance of 238 unique fOTUs representing 21 fungal classes was altered 361 by fungicide treatments across the two crops. Ascomycota (52.9\%) and Basidiomycota (43.3\%)

362 fOTUs made up $96.2 \%$ of the differentially abundant fOTUs. Within Ascomycota, the 363 Dothidiomycetes (28.6\%) and Sordariomycetes (9.66\%) accounted for the largest percentage of 364 fOTUs that were differentially abundant following fungicide treatment (Fig. 3a). These fungi may be 365 expected since many foliar plant pathogens fall within this class of fungi, and fungicides typically 366 target these pathogen groups. Unexpectedly, a large percentage of fOTUs that were differentially 367 abundant included non-target dimorphic clades of fungi that commonly exist as yeasts such as 368 Agaricostilbomycetes, Cystobasidiomycetes, Exobasidiomycetes, Microbotryomycetes, 369 Spiculogleomycetes, Taphrinomycetes, and Tremellomycetes. A total of 83 fOTUs across these 370 classes were significantly different in abundance following the fungicide application in maize or 371 soybean (Fig. 3a). Notably, Tremellomycetes made up the second-largest class of fungi (42 fOTUs, $37217.6 \%$ ) differentially abundant. Of the Tremellomycetes, $57.1 \%$ were concentrated within the 373 Bulleribasidiaceae, accounting for 24 fOTUs that were differentially abundant compared to non374 sprayed control. Twenty-three of the Bulleribasidiaceae significantly decreased in abundance. 375 However, not all yeast fOTUs decreased in abundance. For example, Bulleromyces albus fOTU10 376 increased in relative abundance 4.25 times in soybean conventional management 13-dpf but was not 377 significantly different than the control after 33-dpf. In contrast, two Sporobolomyces fOTUs (fOTU66 378 and fOTU94) increased relative abundance following fungicide application in soybean and remained 379 significantly (7 times) higher in fungicide treated plots than in control plots 33-dpf. Sporobolomyces 380 patagonicus fOTU94 was 4.38 times more abundant in the fungicide treated plots than the control 13381 dpf in the conventional management and remained significantly higher in fungicide sprayed plots 
382 (9.06 times) compared to the control after 33-dpf. Sporobolomyces roseus fOTU66 was 15 times

383 more abundant in the conventionally managed fungicide treated plots 33-dpf. This same increase in 384 Sporobolomyces abundance was not observed in maize.

386 Resilience of the core mycobiome and local extinctions of accessory members. Many of the 387 fOTUs affected by a fungicide application were also part of the core phyllosphere taxa (Table S8). In 388 conventionally managed soybean plots, 22 fOTUs were determined to be core fungal phyllosphere 389 taxa, and the abundances of five of these core members (fOTU 6 Mycosphaerella sp., fOTU 10 390 Tremellales, fOTU 34 Hannaella sp., fOTU 13 Hannaella sp., and fOTU 643 Tilletiopsis sp.) were 391 significantly different following fungicide application. Hannaella sp. (fOTU 13), Hannaella sp. 392 (fOTU 13), and Tilletiopsis sp. (fOTU 643) were also part of the 43 core members of the no-till 393 soybean phyllosphere affected by fungicide application. Of the 40 core members of the 394 conventionally managed maize phyllosphere, the abundance of four Tremellomycetes fOTUs and one 395 unidentified fungal taxon (fOTU 116) were significantly different following fungicide application. 396 These included three yeast taxa that were not members of the soybean core, which included two 397 fOTUs in the genus Filobasidium (fOTU 82 Filobasidium oeirense, and fOTU 97 Filobasidium), one 398 Bullera crocea (fOTU 65), and Vishniacozyma globispora (fOTU 83). Two of these fOTUs (fOTU 39997 Filobasidium and fOTU 65 Bullera crocea) were also core members of the maize phyllosphere in 400 the no-till management that were significantly altered by the fungicide.

$401 \quad$ None of the core members of the phyllosphere taxa became locally extinct following 402 fungicide application in the core microbiome of both crop managements. However, among the taxa 403 whose abundance was significantly altered by the fungicide application in soybean, the no-till 404 management had a $61 \%$ recovery compared to the $34 \%$ recovery of the conventionally managed 405 soybean (Fig. 3b; Table S9). Fourteen fungal OTUs became locally extinct following fungicide 406 application in soybean managed conventionally compared to one in the no-till plots (Fig. 3b). Among 407 the Tremellomycetes fOTUS whose abundances were significantly impacted by fungicide 408 applications, the majority were Bulleribasidiaceae (Fig. 3c). Eighty-two percent of affected 409 Bulleribasidiaceae recovered in no-till managed soybean compared to the $30 \%$ of the conventionally 410 managed plots. No Bulleribasidiaceae taxa became locally extinct in the no-till plots; in contrast, 411 three Bulleribasidiaceae fOTUs were never observed following fungicide application in the 412 conventional management (Figure 3d). The trend of increased recovery was also evident in 
413 Bulleribasidiaceae richness on the last sampling for maize (33-dpf) and soybean (34-dpf) no-till 414 samples (Fig. 3e, 3f). Bulleribasidiaceae in the core of conventional management did not fully 415 recover within the study period (Fig. 3g). Additionally, the Bulleribasidiaceae in the conventional 416 management that were locally extinct following fungicide disturbance occupied less than $50 \%$ 417 samples in non-sprayed plots indicating that local extinctions caused by fungicides affect the rare, 418 non-core members of the community (Fig. $3 \mathrm{~g}$ ). No local extinctions among fungal taxa were detected 419 in maize fungicide treated plots; all impacted taxa recovered.

421 Indirect effects of fungicides on prokaryotes mediated through yeast. Random forest models 422 based on prokaryotic abundance on soybean leaves sprayed with fungicides explained a significant 423 amount of variance $(P<0.001)$ in Bulleribacideaceae richness in the no-till $\left(28.70 \% ; \mathrm{R}^{2}=0.25\right)$ and 424 conventional $\left(43.47 \% ; \mathrm{R}^{2}=0.44\right)$ management (Fig. 4a, 4b). We then extracted the set of OTUs that 425 were the most important for maintaining the model's accuracy in fungicide treated plots. However, 426 there was no evidence $(P \geq 0.05)$ those same taxa were able to predict Bulleribacideaceae richness in 427 control samples indicating the unique effect of the fungicide (Fig. S3). OTUs classified as 428 Sphingomonas, Methylobacterium, and Hymenobacter were the most important for predicting fungal 429 richness in the no-till management (Fig. 4a, 4b). Many taxa from the same genera were important in 430 predicting Bulleribasidiaceae richness in the conventional management system, including the 431 Sphingomonas and Hymenobacter genera (Fig. 4c, 4d). However, other genera were unique by 432 management type, including Methylobacterium for the no-till management system and 433 Pseudokineococcus and Kineococcus in the conventional management system $434 \quad$ Prokaryote OTUs that were important for random forest model accuracy increased in 435 abundance in fungicide treated plots as a response to altering Bulleribacideaceae diversity and were 436 negatively co-associated with Bulleribacideaceae (Fig. 4b, 4d, 4e, 4f). For example, pOTU21 437 Hymenobacter and pOTU1874 Sphingomonas abundance increased as Bulleribacideaceae richness 438 decreased and was negatively co-associated with the Bulleribacideaceae (Fig. 4b, 4e, 4f). 439 Additionally, the cumulative edge weight between pOTU21 Hymenobacter, pOTU1874 440 Sphingomonas, and Bulleribacideaceae significantly changed when sprayed with fungicides in the 441 conventional management, but not always in the no-till management, indicating that a loss in 442 Bulleribacideace diversity can indirectly influence the co-occurrence between fungi and bacteria in 443 different crop management schemes. However, not all co-occurrences between the Bulleribacideaceae 
and prokaryotes were negative, indicating that positive co-occurrences between prokaryotes and fungi in the phyllosphere may shift as well (Fig. 4e, 4f).

\section{Discussion}

To our knowledge, this is the first study to assess the effect of fungicide-imposed disturbance and resiliency under different agricultural management systems. We found that fungicide applications had little direct effect on root fungal or prokaryotic communities but a substantial effect on target and non-target fungal phyllosphere communities and minor indirect effects on prokaryote communities in the phyllosphere. Soil fungi and prokaryotes were also sequenced in soybean, and there was no evidence of fungicidal effects (data not shown). Leveraging the KBS LTER site allowed the direct comparison of long-term crop management impacts to the microbiome without confounding location. Our data demonstrate that the phyllosphere microbiome's resilience depends on the cropping management system, with a greater recovery in the abundance of affected phyllosphere microbiota in long-term no-till compared to annually tilled conventional management. Among the most important results was the commonality in the fungal taxa affected by fungicide treatments, particularly nontargeted groups of phyllosphere yeasts across crops. In maize and soybean, fungi in Dothidiomycetes (target) and Tremellomycetes (non-target) decreased in abundance following fungicide applications, raising questions on the role of Tremellomycete yeasts; specifically, the Bulleribasidiaceae play in phyllosphere microbiomes, and the effects of fungicide used in the absence of disease pressure.

This study observed reductions or local extinctions of these yeasts following fungicide application, which may lead to unintended consequences in the host plant. Phyllosphere yeast communities have received less attention in the literature than prokaryotes (Lindow \& Brandl, 2003). The three Bulleribacideaceae genera observed in this study were Hannaella, Dioszengia, and Vishniacozyma, Dioszengia, and Hannaella have been demonstrated to produce the plant growthpromoting hormone indole acetic acid (IAA), similar to many plant growth-promoting phyllosphere prokaryotes (Sun et al., 2014; Wang et al., 2016). In comparison, Vishniacozyma yeasts have remained understudied but have been isolated from maize kernels (Yurkov \& Kurtzman, 2019). Agler et al. (2016) and Wang et al. (2016) found Dioszegia was a hub taxon important in maintaining fungal-prokaryote interactions by altering prokaryote diversity in the phyllosphere microbiome of Arabidopsis. Importantly, in the absence of disease pressure as observed in this study, our results 
indicate that fungicide applications may affect populations of resident beneficial microbes. However, adverse impacts would be expected to be outweighed if the fungicide mitigates the disease.

Here, we show for the first time that fungicidal impacts on crop microbiomes is dependent on crop management, addressing a knowledge gap that previous studies were unable to address specifically (Karlsson et al., 2014; Sapkota et al., 2015; Knorr et al., 2019). A higher proportion of fOTUs altered by fungicide application in the no-till management system showed improved resilience within the study period, which may be explained by the differences in microbial communities present in the phyllosphere of each management before fungicide applications, as has been demonstrated previously at the KBS LTER site (Longley et al., 2020; Gdanetz \& Trail, 2017). A previous study from the KBS LTER site has demonstrated that aerially dispersed yeasts are enriched in the phyllosphere but also found in lower abundance in belowground plant organs (Gdanetz et al., 2021). Crop residue left from previous seasons can harbor fungi that potentially may act as a source to repopulate the phyllosphere following a disturbance like the phenomenon of pathogens transferring from residues (Sommermann et al., 2018). Yeasts that inhabit the phyllosphere are primarily known to disperse through ballistosporic aerial dispersal, and the reassembly of leaves following fungicides may rely heavily on this spore dispersal mechanism. However, not all yeast taxa in the Bulleribacideaceae have been observed to form ballistocondia in culture (Li et al., 2020), leaving arguably less efficient means of dispersal from insects or through wind and rain (Gilbert, 1980; Starmer et al., 1988). Regardless, locally extinct taxa were not part of the core microbiome regardless of management systems or spore dispersal mechanism, demonstrating a tight relationship between abundance-occupancy and disturbance. These results demonstrate that microbiome resilience is improved in no-till crop management, informs discussion on managing crops for resilience, and demonstrates an ecosystem service provided by no-till agriculture in addition to improved nutrient cycling or preservation of habitats for microorganisms and mesofauna (Murrell, 2017).

In addition to differential impacts by crop management, fungicide applications affected soybean and maize phyllosphere communities differently. These differences may be due to crop, planting year, or fungicide regime. The effect of fungicide was likely reduced in the final sampling of maize due to sampling new leaves not directly sprayed, indicating that any effect would have been through the systemic activity of the fungicide 34 days after, which may have decreased the effect on the microbiome. Another critical difference is that the Delaro ${ }^{\circledR}$ fungicide applied to soybeans in 2018 has two modes of action. Application of fungicides having two different modes of action has been 
505 shown to have a more significant effect on fungal beta diversity than a single mode of action in cereal 506 crops (Sapkota et al., 2015). Although the impact of fungicides varied in magnitude between the two 507 crops, the commonality of off-target impacted taxa between crops and fungicides demonstrates that 508 multiple fungicide products on different crops consistently reduce these taxa. This information can be 509 used to inform decisions on the use of fungicides under low pathogen pressure across multiple crops 510 and cropping systems.

511 Recovery of network complexity is one measure of microbiome resilience. We show that 512 network complexity decreased significantly in the soybean phyllosphere following fungicide 513 treatment. Other studies have demonstrated that agricultural management alters network complexity. 514 However, the functional consequences of these changes were not directly assessed (Banerjee et al., 515 2019; Schmidt et al., 2019). In soils, it has been demonstrated that increases in network complexity 516 are positively correlated with various ecosystem functions and increases in the number of unique 517 functions and functional redundancy (Wagg et al., 2019). The functional consequences of decreases 518 in network complexity remain unexplored in the phyllosphere microbiome. They may provide the 519 rationale for chemical application decisions or novel microbial-based treatments to replace lost taxa. $520 \quad$ Notably, fungicide application altered co-occurrences between phyllosphere fungi and 521 prokaryotes, demonstrating the indirect effects of fungicide applications through the loss in the 522 diversity of Bulleribacideaceae. In support of random forest results, many of the same prokaryotes 523 identified from networks as having changes in cumulative mean edge weight were identified by 524 random forest as predicting Bulleribasidiaceae richness. Disturbance can change 525 cooperation/competition dynamics, and a high level of disturbance can reduce cooperation 526 (Brockhurst et al., 2007; 2010). In our study, the cumulative mean edge weight between most 527 phyllosphere prokaryotes and Bulleribasidiaceae became more positive, indicating fewer negative 528 associations between a particular bacterium and the Bulleribasidiaceae. However, there were 529 exceptions where cumulative edge weights, positive before spray became neutral, likely due to the 530 disappearance of some fungal taxa from samples following fungicide application, and therefore the 531 disappearance of any associations. However, loss of negative correlations may also be due to reduced 532 competition between phyllosphere prokaryotes and Bulleribasidiaceae as more niche space is 533 available to phyllosphere prokaryotes following fungicide application.

534 Shifts in correlations between Bulleribasidiaceae and phyllosphere prokaryotes are of interest 535 due to the unique physiology of many phyllosphere prokaryotes as it relates to plant health. 
536 Methylobacterium spp. have been demonstrated to be abundant in plants' phyllosphere and have the

537 genes to produce plant growth-promoting auxins and UVA-absorbing compounds (Kwak et al., 2014;

538 Yoshida et al., 2017). Hymenobacter sp., Methylobacterium sp., and Sphingomonas sp. are core

539 phyllosphere members in switchgrass (Grady et al., 2019) and are highly abundant in the Arabidopsis

540 phyllosphere (Delmotte et al., 2009).

541 A comprehensive view of the phyllosphere organisms is needed to understand microbiome

542 functioning and plant health. This research demonstrates that foliar fungicide treatments alter

543 phyllosphere microbiomes in maize and soybean, and non-target Bulleribacideaceae yeasts were

544 negatively impacted in soybean and maize phyllospheres. Microbiome complexity was altered

545 partially by decreasing co-occurrence between Bulleribacideaceae yeasts and dominant phyllosphere

546 prokaryote taxa, demonstrating indirect effects of fungicide applications mediated through the

547 presence of these yeasts. Further, these data support our hypothesis that the recovery of the

548 phyllosphere microbiome differed by tilling management. Together, these results improve our

549 understanding of fungicide impacts on crop microbiomes and their recovery in different

550 managements and inform their rational use to maintain efficacy and intended impacts across different

551 cropping systems.

552

\section{Acknowledgments}

554 This work was supported by the NIFA grant MICL08541 from the USDA National Institute of Food 555 and Agriculture to GB, FT, and MC and USDA MICL02416 to GB. Support for this research was 556 also provided by the NSF Long-term Ecological Research Program (DEB 1832042) at the Kellogg 557 Biological Station and by Michigan State University AgBioResearch. We would also like to thank 558 Elizabeth Gall for field and lab assistance. We would like to thank Alex Witte for help with applying 559 the fungicides.

560

\section{Author Contributions}

562 ZAN and RL contributed equally to this work. MIC, FT, and GB designed experiments. MIC applied 563 fungicides. MIC, FT, GB, and RL collected samples. ZAN and RL prepared amplicon libraries and 564 generated sequencing data. ZAN, RL, GMNB, and GB wrote and edited the manuscript, with 565 contributions from MIC and FT. ZAN, RL, GMNB processed sequencing data and analyzed data. 566 ZAN generated figures. 
Data availability. Raw sequences for soybean microbial communities used to create figures and tables in this study are available in the NCBI SRA database under the following accession numbers: PRJNA603199 and PRJNA603207. Sequences which were produced on the same Miseq runs and used to remove contaminants are available in PRJNA603147. Raw sequences for maize microbial communities are available under the following accession numbers: PRJNA739465 and PRJNA739759. Code to generate figures and tables are located on GitHub at https://github.com/noelzach/FungicidePulseDisturbance

\section{References}

Abarenkov K, Zirk A, Piirmann T, Pöhönen R, Ivanov F, Nilsson RH, Kõljalg U. 2020. UNITE general FASTA release for eukaryotes. Version 8.2 04.02.2020. UNITE Community.

Agler MT, Ruhe J, Kroll S, Morhenn C, Kim S-T, Weigel D, Kemen EM. 2016. Microbial Hub

Taxa Link Host and Abiotic Factors to Plant Microbiome Variation. PLoS biology 14: e1002352.

Anderson MJ, Willis TJ. 2003. CANONICAL ANALYSIS OF PRINCIPAL COORDINATES: A USEFUL METHOD OF CONSTRAINED ORDINATION FOR ECOLOGY. Ecology 84: 511-525.

Augusto J, Brenneman TB. 2012. Assessing Systemicity of Peanut Fungicides Through Bioassay of Plant Tissues with Sclerotium rolfsii. Plant disease 96: 330-337.

Banerjee S, Walder F, Büchi L, Meyer M, Held AY, Gattinger A, Keller T, Charles R, van der

Heijden MGA. 2019. Agricultural intensification reduces microbial network complexity and the abundance of keystone taxa in roots. The ISME journal 13: 1722-1736.

593 Bowsher AW, Benucci GMN, Bonito G, Shade A. 2020. Seasonal Dynamics of Core Fungi in the 594 Switchgrass Phyllosphere, and Co-Occurrence with Leaf Bacteria. Phytobiomes Journal: PBIOMES59507.

Brockhurst MA, Buckling A, Gardner A. 2007. Cooperation peaks at intermediate disturbance. 
598 Current biology: $C B$ 17: 761-765.

600 Brockhurst MA, Habets MGJL, Libberton B, Buckling A, Gardner A. 2010. Ecological drivers 601 of the evolution of public-goods cooperation in bacteria. Ecology 91: 334-340.

603 Cadez N, Zupan J, Raspor P. 2010. The effect of fungicides on yeast communities associated with 604 grape berries. FEMS yeast research 10: 619-630.

Caporaso JG, Kuczynski J, Stombaugh J, Bittinger K, Bushman FD, Costello EK, Fierer N, 607 Peña AG, Goodrich JK, Gordon JI, et al. 2010. QIIME allows analysis of high-throughput 608 community sequencing data. Nature methods 7: 335-336.

609

Caporaso JG, Lauber CL, Walters WA, Berg-Lyons D, Lozupone CA, Turnbaugh PJ, Fierer N, Knight R. 2011. Global patterns of $16 \mathrm{~S}$ rRNA diversity at a depth of millions of sequences per sample. Proceedings of the National Academy of Sciences 108: 4516-4522.

613

614 Claassen R, Bowman M, McFadden J, Smith D, Wallander S. 2018. Tillage intensity and 615 conservation cropping in the United States.

617 Cobban A, Edgcomb VP, Burgaud G, Repeta D, Leadbetter ER. 2016. Revisiting the pink-red 618 pigmented basidiomycete mirror yeast of the phyllosphere. MicrobiologyOpen 5: 846-855. identification and removal of contaminant sequences in marker-gene and metagenomics data. Microbiome 6: 226. grapevine downy mildew. Molecular ecology 26: 1936-1951. 
629 Vorholt JA. 2009. Community proteogenomics reveals insights into the physiology of phyllosphere 630 bacteria. Proceedings of the National Academy of Sciences of the United States of America 106:

631 16428-16433.

632

633 Dickinson CH, Wallace B. 1976. Effects of late applications of foliar fungicides on activity of 634 micro-organisms on winter wheat flag leaves. Transactions of the British Mycological Society 67: $635 \quad 103-112$.

637 Edgar RC. 2010. Search and clustering orders of magnitude faster than BLAST. Bioinformatics 26: $6382460-2461$.

640 Edgar RC. 2013. UPARSE: highly accurate OTU sequences from microbial amplicon reads. Nature 641 methods 10: 996-998.

642

643 Fehr WR, Caviness CE, Burmood DT, Pennington JS. 1971. Stage of development descriptions 644 for soybeans, Glycine max (L.) Merrill 1. Crop science 11: 929-931.

646 Fonseca Á, Inácio J. 2006. Phylloplane Yeasts. In: Péter G, Rosa C, eds. Biodiversity and 647 Ecophysiology of Yeasts. Berlin, Heidelberg: Springer Berlin Heidelberg, 263-301.

\section{Fournier B, Dos Santos SP, Gustavsen JA, Imfeld G, Lamy F, Mitchell EAD, Mota M, Noll D,}

Planchamp C, Heger TJ. 2020. Impact of a synthetic fungicide (fosetyl-Al and propamocarbhydrochloride) and a biopesticide (Clonostachys rosea) on soil bacterial, fungal, and protist communities. Science of The Total Environment 738: 139635.

654 Freimoser FM, Rueda-Mejia MP, Tilocca B, Migheli Q. 2019. Biocontrol yeasts: mechanisms and 655 applications. World journal of microbiology \& biotechnology 35: 154.

Gardes M, Bruns TD. 1993. ITS primers with enhanced specificity for basidiomycetes--application 658 to the identification of mycorrhizae and rusts. Molecular ecology 2: 113-118. 
660 Gdanetz K, Benucci GMN, Vande Pol N, Bonito G. 2017. CONSTAX: a tool for improved 661 taxonomic resolution of environmental fungal ITS sequences. BMC bioinformatics 18: 538. 662 663 Gdanetz K, Trail F. 2017. The Wheat Microbiome Under Four Management Strategies, and 664 Potential for Endophytes in Disease Protection. Phytobiomes Journal 1: 158-168.

665

Gdanetz K, Noel Z, Trail F. 2021. Influence of Plant Host and Organ, Management Strategy, and 667 Spore Traits on Microbiome Composition. Phytobiomes Journal 5: 202-219

668

Gilbert DG. 1980. Dispersal of yeasts and bacteria by Drosophila in a temperate forest. Oecologia 670 46: $135-137$.

671

Glasby TM, Underwood AJ. 1996. Sampling to differentiate between pulse and press perturbations.

Environmental monitoring and assessment 42: 241-252.

674

Grady KL, Sorensen JW, Stopnisek N, Guittar J, Shade A. 2019. Assembly and seasonality of 676 core phyllosphere microbiota on perennial biofuel crops. Nature communications 10: 4135.

677

Hahn M. 2014. The rising threat of fungicide resistance in plant pathogenic fungi: Botrytis as a case study. Journal of chemical biology 7: 133-141.

680

Imfeld G, Vuilleumier S. 2012. Measuring the effects of pesticides on bacterial communities in soil: 682 A critical review. European journal of soil biology 49: 22-30.

683

Jari Oksanen FGB, Friendly M, Kindt R, Legendre P, McGlinn D, Minchin PR, O'Hara RB, 685 Simpson GL, Solymos P, Stevens MHH, et al. 2018. Vegan: community ecology package. $R$ 686 package version 2.

687

Kandel YR, Hunt C, Ames K, Arneson N, Bradley CA, Byamukama E, Byrne A, Chilvers MI, 689 Giesler LJ, Halvorson J, et al. 2021. Meta-Analysis of Soybean Yield Response to Foliar 690 Fungicides Evaluated from 2005 to 2018 in the United States and Canada. Plant disease: 
PDIS07201578RE.

Karlsson I, Friberg H, Steinberg C, Persson P. 2014. Fungicide effects on fungal community composition in the wheat phyllosphere. PloS one 9: e111786.

695

Knorr K, Jørgensen LN, Nicolaisen M. 2019. Fungicides have complex effects on the wheat phyllosphere mycobiome. PloS one 14: e0213176.

698

Kursa MB, Rudnicki WR. 2010. Feature Selection with the Boruta Package. Journal of Statistical

Software 36: 1-13.

701

702

Kurtz ZD, Müller CL, Miraldi ER, Littman DR, Blaser MJ, Bonneau RA. 2015. Sparse and

703

compositionally robust inference of microbial ecological networks. PLoS computational biology 11:

704 e1004226.

705

706

Kwak M-J, Jeong H, Madhaiyan M, Lee Y, Sa T-M, Oh TK, Kim JF. 2014. Genome information

707 of Methylobacterium oryzae, a plant-probiotic methylotroph in the phyllosphere. PloS one 9:

708 e106704.

709

710

Landers TF, Cohen B, Wittum TE, Larson EL. 2012. A review of antibiotic use in food animals: perspective, policy, and potential. Public health reports 127: 4-22.

712

Latin R. 2017. A Practical Guide to Turfgrass Fungicides.

714

715 Li A-H., Yuan, F.-X., Groenewald, M., Bensch, K., Yurkov, A. M., Li, K., Han, P.-J., Guo, L.716 D., Aime, M. C., Sampaio, J. P., Jindamorakot, S., Turchetti, B., Inacio, J., Fungsin, B., Wang, 717 Q.-M., Bai, F.-Y. 2020. Diversity and phylogeny of basidiomycetous yeasts from plant leaves and soil: Proposal of two new orders, three new families, eight new genera and one hundred and seven new species. Studies in Mycology 96. https://doi.org/10.1016/j.simyco.2020.01.002</div>

Liaw A, Wiener M. 2002. Classification and regression by randomForest. $R$ news 2: 18-22. 
723 Liber J, Bonito G, Benucci GMN. 2021. CONSTAX2: Improved taxonomic classification of

724 environmental DNA markers. Bioinformatics, https://doi.org/10.1093/bioinformatics/btab347

725

726

Lindow SE, Brandl MT. 2003. Microbiology of the phyllosphere. Applied and environmental

727

microbiology 69: 1875-1883.

728

729

Longley R, Noel ZA, Benucci GMN, Chilvers MI, Trail F, Bonito G. 2020. Crop Management

Impacts the Soybean (Glycine max) Microbiome. Frontiers in Microbiology 11.

Lundberg DS, Yourstone S, Mieczkowski P, Jones CD, Dangl J. 2013. Practical innovations for

high-throughput amplicon sequencing. Nat. Methods 10:999-1002.

734

Mandal S, Van Treuren W, White RA, Eggesbo M, Knight R, Peddada SD. 2015. Analysis of composition of microbiomes: a novel method for studying microbial composition. Microbial ecology in health and disease 26: 27663.

738

Martin M. 2011. Cutadapt removes adapter sequences from high-throughput sequencing reads.

EMBnet.journal 17: 10.

\section{McDonald MC, Renkin M, Spackman M, Orchard B, Croll D, Solomon PS, Milgate A. 2019.}

Rapid parallel evolution of azole fungicide resistance in Australian populations of the wheat pathogen

Zymoseptoria tritici. Applied and environmental microbiology 85.

McMurdie PJ, Holmes S. 2013. phyloseq: an R package for reproducible interactive analysis and graphics of microbiome census data. PloS one 8: e61217.

Murrell EG. 2017. Can agricultural practices that mitigate or improve crop resilience to climate change also manage crop pests? Current Opinion in Insect Science 23: 81-88. 
marker-gene surveys. Nature methods 10: 1200-1202.

Perazzolli M, Antonielli L, Storari M, Puopolo G, Pancher M, Giovannini O, Pindo M, Pertot I.

2014. Resilience of the natural phyllosphere microbiota of the grapevine to chemical and biological

pesticides. Applied and environmental microbiology 80: 3585-3596.

758

759

\section{Quast C, Pruesse E, Yilmaz P, Gerken J, Schweer T, Yarza P, Peplies J, Glöckner FO. 2013.}

760 The SILVA ribosomal RNA gene database project: improved data processing and web-based tools.

Nucleic acids research 41: D590-6.

762

R Core Team. 2020. $R$ : A Language and Environment for Statistical Computing. Vienna: R

Foundation for Statistical Computing.

765

766

Riat A, Plojoux J, Gindro K, Schrenzel J, Sanglard D. 2018. Azole Resistance of Environmental 767 and Clinical Aspergillus fumigatus Isolates from Switzerland. Antimicrobial agents and chemotherapy 62.

769

770

Ripple WJ, Beschta RL. 2005. Linking Wolves and Plants: Aldo Leopold on Trophic Cascades.

772

773

Robertson GP, Hamilton SK. 2015. Long-term ecological research at the Kellogg Biological Station 774 BioScience 55: 613. $1-32$.

Rykiel EJ. 1985. Towards a definition of ecological disturbance. Austral ecology 10: 361-365.

Sahasrabudhe S, Motter AE. 2011. Rescuing ecosystems from extinction cascades through

780 
Schaeffer RN, Mei YZ, Andicoechea J, Manson JS, Irwin RE. 2017a. Consequences of a nectar yeast for pollinator preference and performance. Functional ecology 31: 613-621.

786

Schaeffer RN, Vannette RL, Brittain C, Williams NM, Fukami T. 2017b. Non-target effects of fungicides on nectar-inhabiting fungi of almond flowers. Environmental microbiology reports 9: 7984.

Schmidt JE, Kent AD, Brisson VL, Gaudin ACM. 2019. Agricultural management and plant selection interactively affect rhizosphere microbial community structure and nitrogen cycling. Microbiome 7: 146.

Shade A, Peter H, Allison SD, Baho DL, Berga M, Bürgmann H, Huber DH, Langenheder S,

Lennon JT, Martiny JBH, et al. 2012. Fundamentals of microbial community resistance and

resilience. Frontiers in microbiology 3: 417.

798

Shade A, Stopnisek N. 2019. Abundance-occupancy distributions to prioritize plant core microbiome membership. Current opinion in microbiology 49: 50-58.

801

803 Schellenberg I. 2018. Fungal community profiles in agricultural soils of a long-term field trial under different tillage, fertilization and crop rotation conditions analyzed by high-throughput ITS-amplicon sequencing. PLOS ONE 13: e0195345.

Southwell RJ, Brown JF, Welsby SM. 1999. Microbial interactions on the phylloplane of wheat and 808 barley after applications of mancozeb and triadimefon. Australasian Plant Pathology 28: 139.

Starmer WT, Peris F \& Fontdevila A. 1988. The transmission of yeasts by Drosophila buzzatii 811 during courtship and mating. Animal Behaviour 36: 1691-1695.

813 Sullivan TO, Sullivan DS. 2002. Vegetation management and ecosystem disturbance: impact of 814 glyphosate herbicide on plant and animal diversity in terrestrial systems. Environmental Reviews 
$815 \quad 11: 37-59$.

816

817 Sulowicz S, Cycoń M, Piotrowska-Seget Z. 2016. Non-target impact of fungicide tetraconazole on

818 microbial communities in soils with different agricultural management. Ecotoxicology 25: 1047-

8191060.

820

821 Sun P-F, Fang W-T, Shin L-Y, Wei J-Y, Fu S-F, Chou J-Y. 2014. Indole-3-acetic acid-producing 822 yeasts in the phyllosphere of the carnivorous plant Drosera indica L. PloS one 9: e114196.

823 Tilman D. 1999. The Ecological Consequences of Changes in Biodiversity: A Search for General 824 Principles. Ecology 80: 1455-1474.

825

826 Verweij PE, Snelders E, Kema GHJ, Mellado E, Melchers WJG. 2009. Azole resistance in 827 Aspergillus fumigatus: a side-effect of environmental fungicide use? The Lancet infectious diseases 828 9: 789-795.

829

830 Wagg C, Schlaeppi K, Banerjee S, Kuramae EE, van der Heijden MGA. 2019. Fungal-bacterial 831 diversity and microbiome complexity predict ecosystem functioning. Nature communications 10: 8324841.

833 Wang K, Sipilä TP, Overmyer K. 2016. The isolation and characterization of resident yeasts from 834 the phylloplane of Arabidopsis thaliana. Scientific reports 6: 39403.

835

836 Wickham H. 2009. ggplot2: Elegant Graphics for Data Analysis. Springer Science \& Business 837 Media.

838

839 Wise K, Mueller D. 2011. Are fungicides no longer just for fungi? An analysis of foliar fungicide 840 use in corn. APSnet Features. doi $\mathbf{1 0}$.

841

842 Yoshida S, Hiradate S, Koitabashi M, Kamo T, Tsushima S. 2017. Phyllosphere

843 Methylobacterium bacteria contain UVA-absorbing compounds. Journal of photochemistry and 844 photobiology. B, Biology 167: 168-175. 
846 Yurkov AM, Kurtzman CP. 2019. Three new species of Tremellomycetes isolated from maize and 847 northern wild rice. FEMS yeast research 19.

848

849 Zhang J, Liu Y-X, Zhang N, Hu B, Jin T, Xu H, Qin Y, Yan P, Zhang X, Guo X, et al. 2019.

850 NRT1.1B is associated with root microbiota composition and nitrogen use in field-grown rice. Nature 851 Biotechnology 37: 676-684.

852

853 Zhou J, Deng Y, Luo F, He Z, Tu Q, Zhi X. 2010. Functional Molecular Ecological Networks. 854 mBio 1 .

855

Zubrod JP, Bundschuh M, Arts G, Brühl CA, Imfeld G, Knäbel A, Payraudeau S, Rasmussen

JJ, Rohr J, Scharmüller A, et al. 2019. Fungicides: An Overlooked Pesticide Class? Environmental

858

science \& technology 53: 3347-3365.

859

860

Figures and Tables

861

862 Fig. 1 Effects of fungicides on fungal leaf composition in maize and soybean. A separate analysis 863 was conducted for soybean (a) 0- (b) 13- or (c) 33-days post fungicide application or maize (d) 0- (e) 864 9- or (f) 34-days post fungicide application and days post fungicide application (dpf) since there was a 865 significant interaction between dpf and fungicide. Constrained analysis of principal coordinates (CAP) 866 analyses was constrained by the effect of fungicide while partialling out the effect of treatment. The 867 percentage of total variation due to fungicide is expressed above the plot. The significance was 868 determined based on 1000 permutations.

869

870 Fig. 2 Fungicides alter soybean network complexity. A microbial co-occurrence network was 871 constructed using taxa with a mean relative abundance greater than $1^{-5}$ and present in greater than $5 \%$ 872 of samples. Subnetworks were generated for each sample based on the OTUs present within those 873 samples, and each point represents a subnetwork. (a) Network complexity (i.e., linkage density) and 874 (b) number of edges were then calculated for each subnetwork. Comparisons are based on Wilcox 875 ranked sign tests for soybean conventional management and no-till. Asterisks indicate the level of 876 significance; $*=\mathrm{p} \leq 0.05, * *=\mathrm{p} \leq 0.01, * * *=\mathrm{p} \leq 0.001$ 
878 Fig. 3 Management scheme alters the recovery dynamics of phyllosphere fungi following

879 fungicide treatment. The composition of fungal operational taxonomic units (OTUs) significantly

880 different in abundance, as indicated with analysis of compositions of microbiomes (ANCOM)

881 analysis $(\mathrm{n}=12)$. (a) Composition of fOTUs whose abundance was significantly different following a

882 fungicide disturbance. Bars below zero indicate the fOTU decreased in abundance, whereas bars

883 above zero indicate the fOTU increased in abundance. (b) Recovery of fungi in soybean leaf samples

884 in conventional (conv.) or no-till management. (c) Composition of fOTUs within the

885 Tremellomycetes whose abundance was significantly altered following a fungicide disturbance. (d)

886 Recovery dynamics of Tremellomycetes fOTUs following a fungicide disturbance in conv. or no-till

887 (e) soybean or (f) maize plots subjected to a fungicide treatment compared to non-sprayed control

888 plots. (g) Abundance occupancy relationship with the recovery dynamics of the Bulleribasidiaceae

889 fOTUs significantly affected by the fungicide treatment. Asterisks indicate the level of significance; * $890=\mathrm{p} \leq 0.05, * *=\mathrm{p} \leq 0.01, * * *=\mathrm{p} \leq 0.001$

891

892 Fig. 4 Indirect effects of fungicides on prokaryotic communities from altered

893 Bulleribacideaceae diversity on soybean leaves. Relationship of observed versus predicted

894 Bulleribasidiaceae richness in conventional (a) or no-till (c) from random forest models using

895 prokaryote OTU abundance in fungicide treated plots. The most important $(P<0.05)$ prokaryote

896 OTUs for random forest model accuracy in fungicide treated conventional (b) or no-till (d). The

897 cumulative mean edge weight calculated from each sub-network of a meta-network of

898 Bulleribacidaceae edges between Sphinogomonas, Hymenobacter, or Methylobacterium OTUs and

899 alterations to co-occurrence strength with and without fungicides under (e) conventional and (f) no-

900 till crop management.

901

\section{Supplemental Information}

903

904 Fig. S1. Rarefaction curves for each sample sequenced in this study for (a) fungi and (b) prokaryotes

905 in soybean or maize leaves and roots. The dashed line represents the median sequence depth.

906

907 Fig. S2. Fungicidal effects on the richness of different fungal classes in soybean and maize 
908 phyllosphere. Black dots are control yellow dots are fungicide samples. Asterisks indicate the level of

909 significance; $*=\mathrm{p} \leq 0.05, * *=\mathrm{p} \leq 0.01, * * *=\mathrm{p} \leq 0.001$

910

911 Fig. S3. Random forest models percent explained variance, error, and overall model significance

912 (permutations $=999$ ) for (a) conventional management treated with fungicides, (b) no-till treated with

913 fungicides, (c) conventional management control, and (d) no-till control.

914

915 Table S1. Three-step amplicon library preparation reagents and PCR master mixes

916

917 Table S2. Primers used for amplicon library preparation

918

919 Table S3. Cycling conditions for PCR in library preparation for fungi and prokaryotes

920

921 Table S4. Permutational multivariate analysis of variance for fungi in maize or soybean in roots or

922 leaves before and after fungicide application

923

924 Table S5. Permutational multivariate analysis of variance for prokaryotes in maize or soybean in

925 roots or leaves before and after fungicide application

926

927 Table S6. Effects of fungicide on maize and soybean leaf fungal composition

928

929

Table S7. Differentially abundant phyllosphere fungal OTUs by fungicide treatment

930

931 Table S8. Core members of the soybean or maize phyllosphere in no-till and conventional

932 management

933

934 Table S9. Recovery status of fungicide-impacted soybean phyllosphere fungal OTUs

935

936

937

938

939

940 
(a) Soybean Leaf - $0-d p f$

$2 \%$ of total variation $(\mathrm{Cl}=1 \%, 4 \%) ; \mathrm{P}=0.51$

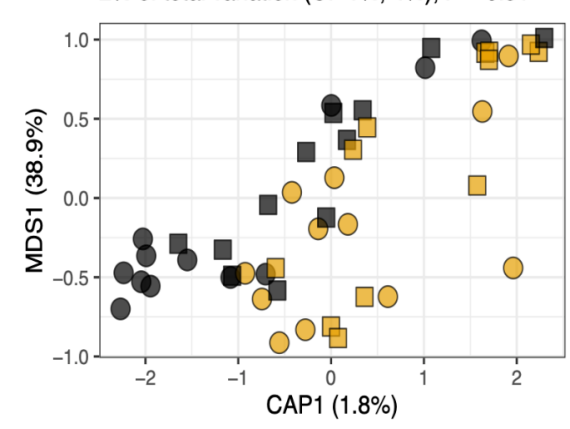

(d) Corn Leaf - 0-dpf

$3 \%$ of total variation $(\mathrm{Cl}=2 \%, 5 \%) ; \mathrm{P}=0.075$

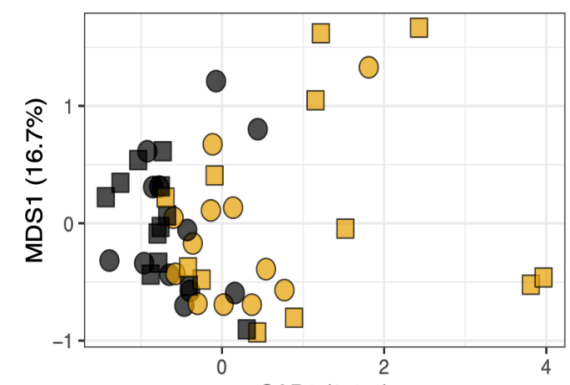

Control $\bigcirc$ Fungicide

(b) Soybean Leaf - 13-dpf

$12 \%$ of total variation $(\mathrm{Cl}=6 \%, 22 \%) ; \mathrm{P}<0.001$

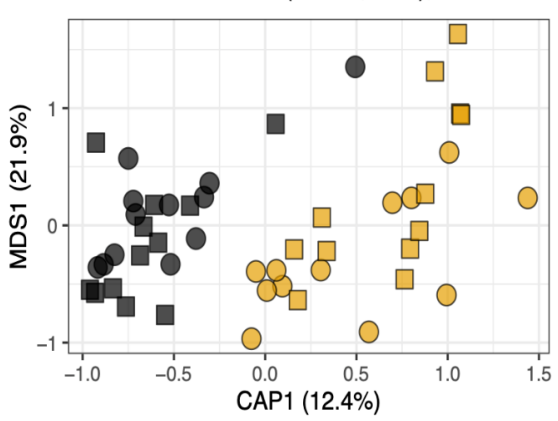

(e) Corn Leaf - 9-dpf

$8 \%$ of total variation $(\mathrm{Cl}=5 \%, 13 \%) ; \mathrm{P}<0.001$

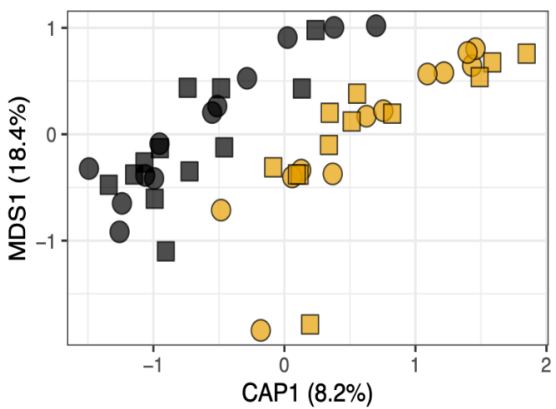

(c) Soybean Leaf - 33-dpf

$11 \%$ of total variation $(\mathrm{Cl}=6 \%, 18 \%) ; \mathrm{P}<0.001$

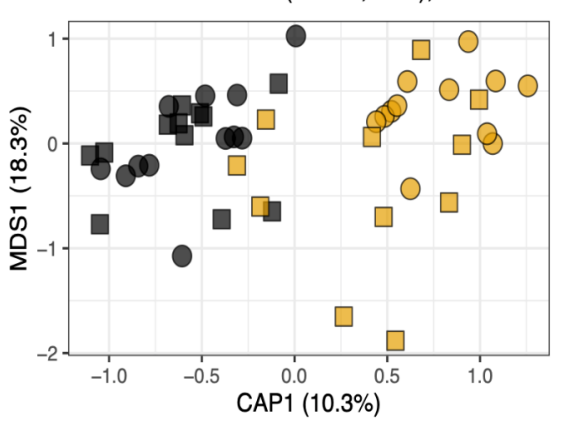

(f) Corn Leaf - 34-dpf $3 \%$ of total variation $(\mathrm{Cl}=2 \%, 6 \%) ; \mathrm{P}=0.078$

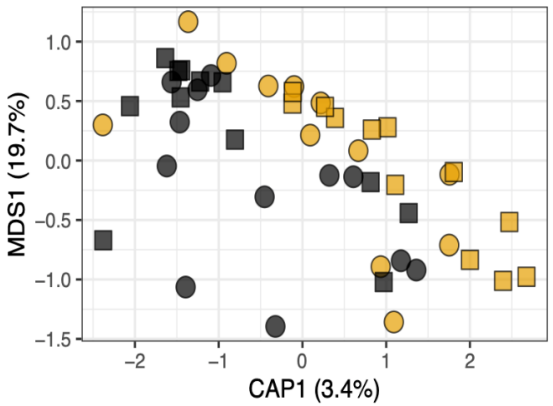

942

943

944

945

946

947

948

949

950

951

952

953

954

Fig. 1 Effects of fungicides on fungal leaf composition in maize and soybean. Separate analyses

were conducted for soybean (a) 0- (b) 13- or (c) 33-days post fungicide application or maize (d) 0- (e)

9- or (f) 34-days post fungicide application and days post fungicide application (dpf) since there was a significant interaction between dpf and fungicide. Constrained analysis of principal coordinates (CAP) analyses was constrained by the effect of fungicide while partialling out the effect of treatment. The percentage of total variation due to fungicide is expressed above the plot. The significance was determined based on 1000 permutations. 
(a)

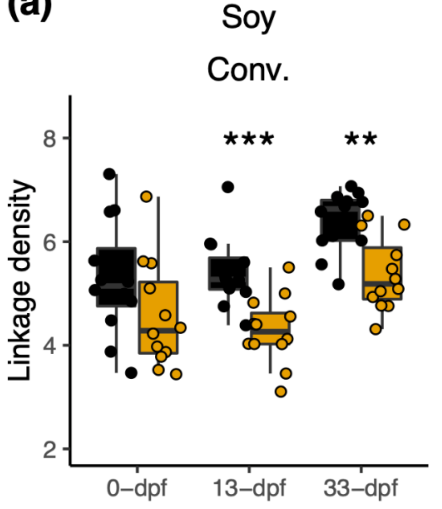

Control 店 Fungicide

(b)

$$
\text { Soy }
$$

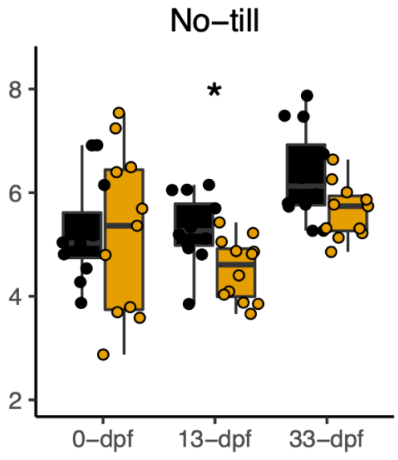

Soy

Conv.
Soy

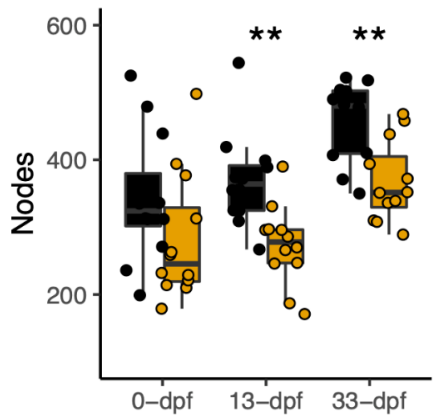

No-till

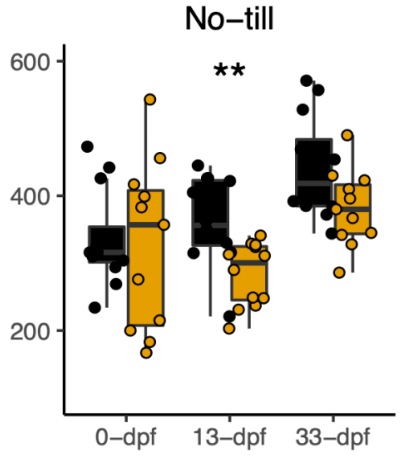

957 constructed using taxa with a mean relative abundance greater than 1-5 and present in greater than 5 $958 \%$ of samples. Subnetworks were created for each sample based on the OTUs present, and each point 959 represents a subnetwork. (a) Network complexity (i.e., linkage density) and (b) number of edges were 960 then calculated for each subnetwork. Comparisons are based on Wilcox ranked sign tests for soybean 961 conventional management and no-till. Asterisks indicate the level of significance; $*=p \leq 0.05, * *=p$ $962 \leq 0.01, * * *=\mathrm{p} \leq 0.001$ 
(a) Fungi

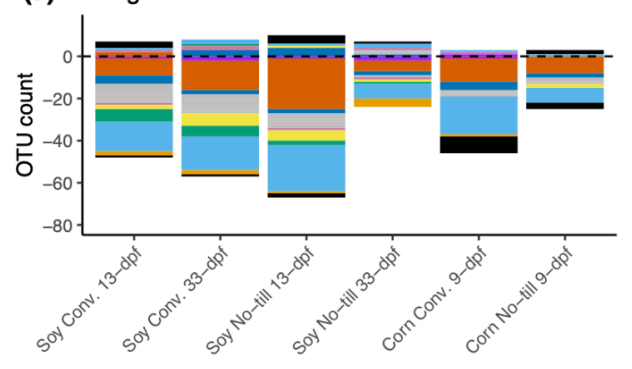

(b) Fungi-Soybean

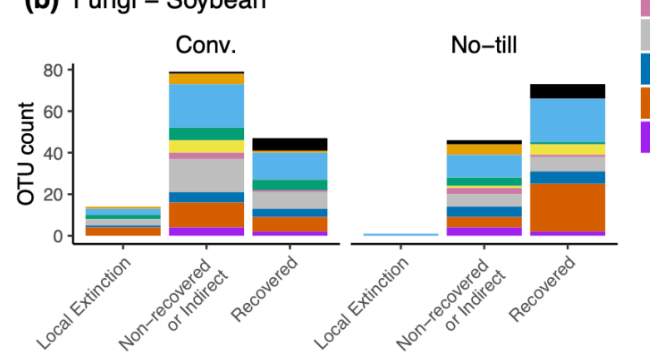

(c) Tremellomycetes

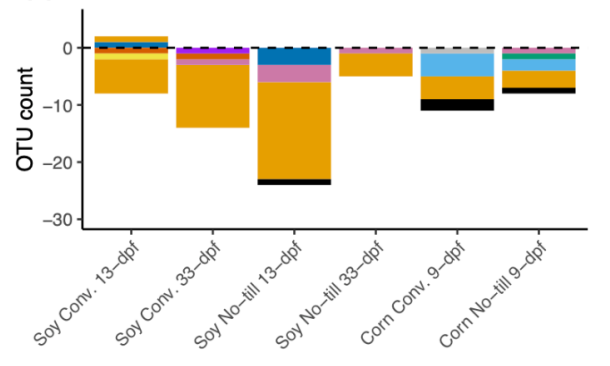

(d) Tremellomycetes - Soybean
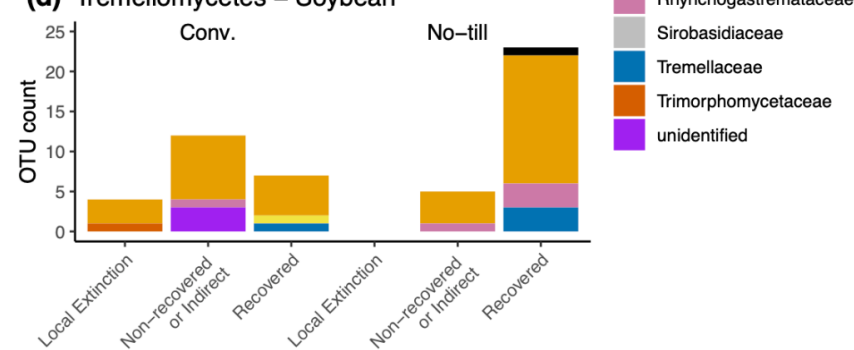

Bulleracea

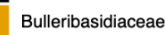

Filobasidiaceae

Incertae_sedis

Mrakiaceae

Rhynchogastremataceae

unidentified (e) Soybean

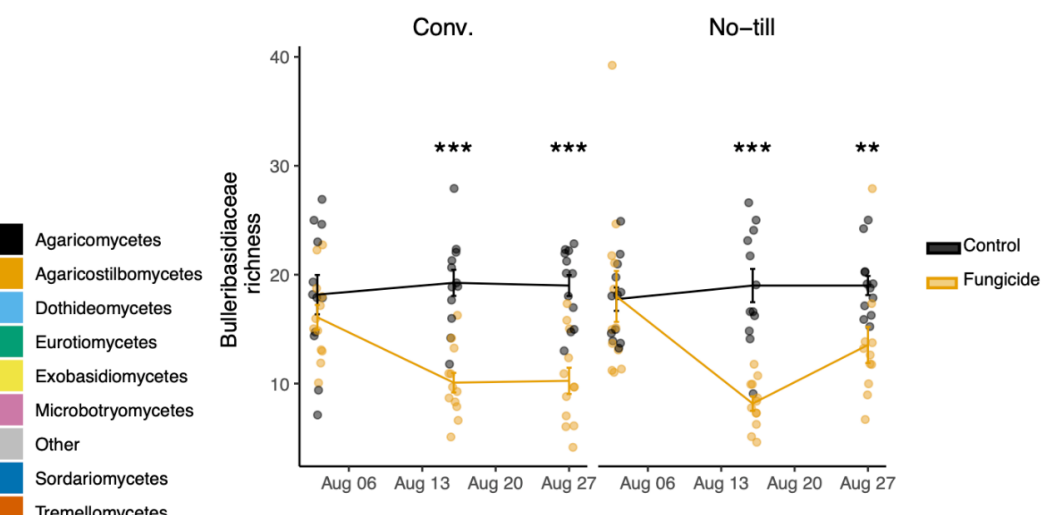

(f) Corn

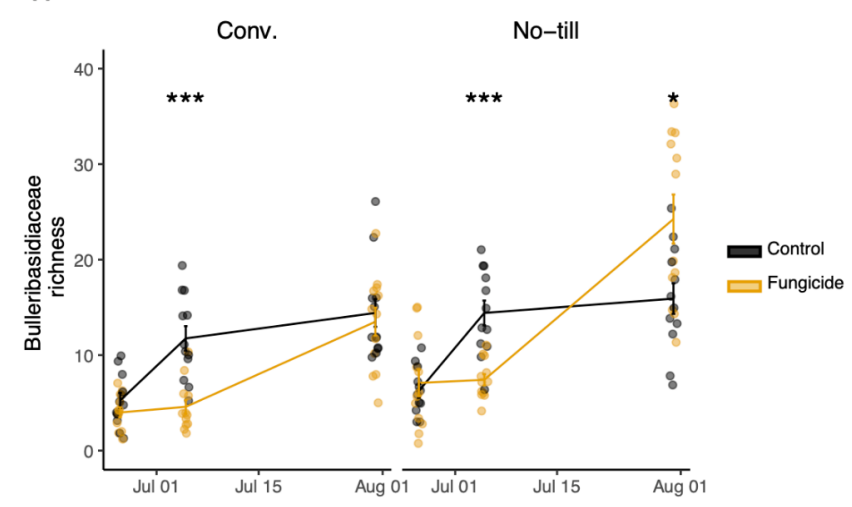

(g) Soybean

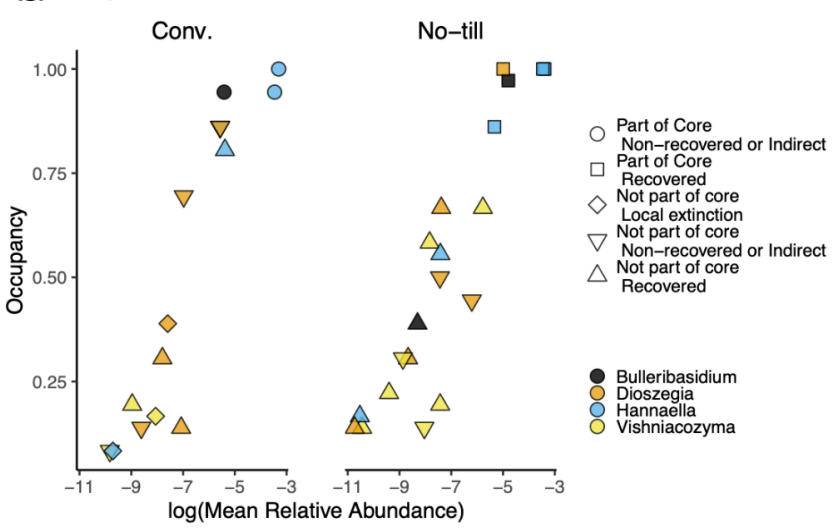

963

964

965

966

967

968

969

970

\section{Fig. 3 Management scheme alters the recovery dynamics of phyllosphere fungi following}

fungicide treatment. The composition of fungal operational taxonomic units (OTUs) significantly

different in abundance, as indicated with analysis of compositions of microbiomes (ANCOM)

analysis $(n=12)$. (a) Composition of fOTUs whose abundance was significantly different following a fungicide disturbance. Bars below zero indicate the fOTU decreased in abundance, whereas bars above zero indicate the fOTU increased in abundance. (b) Recovery of fungi in soybean leaf samples in conventional (conv.) or no-till management. (c) Composition of fOTUs within the 
971 Tremellomycetes whose abundance was significantly altered following a fungicide disturbance. (d) 972 Recovery dynamics of Tremellomycetes fOTUs following a fungicide disturbance in conv. or no-till 973 (e) soybean or (f) maize plots subjected to a fungicide treatment compared to non-sprayed control 974 plots. (g) Abundance occupancy relationship with the recovery dynamics of the Bulleribasidiaceae 975 fOTUs significantly affected by the fungicide treatment. Asterisks indicate the level of significance; * $976=\mathrm{p} \leq 0.05, * *=\mathrm{p} \leq 0.01, * * *=\mathrm{p} \leq 0.001$ 
bioRxiv preprint doi: https://doi.org/10.1101/2021.09.20.461135; this version posted September 21, 2021. The copyright holder for this preprint (which was not certified by peer review) is the author/funder, who has granted bioRxiv a license to display the preprint in perpetuity. It is made available under aCC-BY-NC-ND 4.0 International license.

977

978

979

980

981

982

983

984

985

986

987

988

989

990

991

992

993

994

995

996

997

998

999

1000

1001

1002

1003

1004

1005

1006 (a) Bulleribasidiaceae
richness conv.

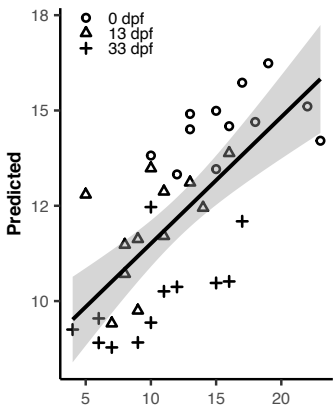

Observed

(c) Bulleribasidiaceae

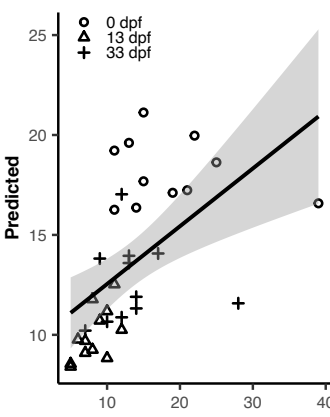

Observed (b) OTU importance

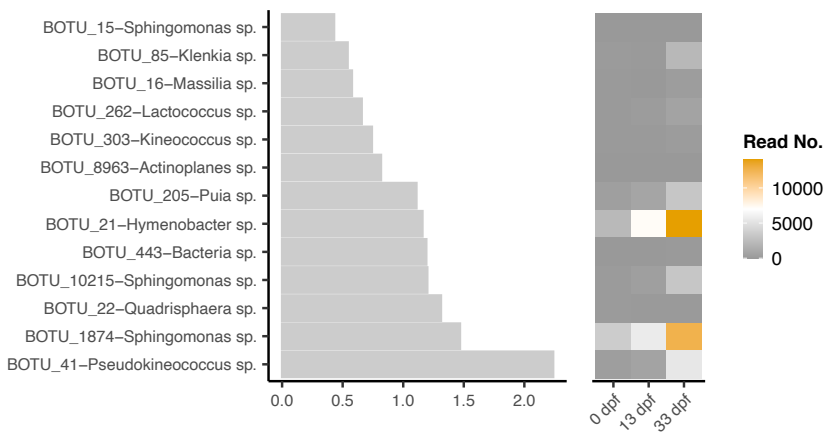

(d)

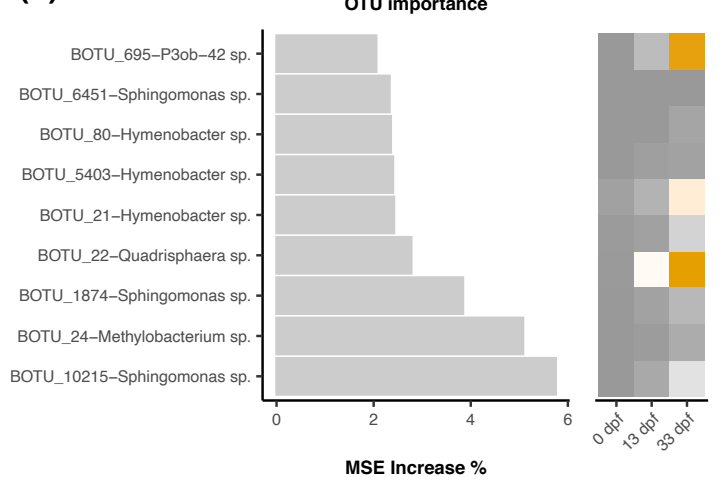

Read No.

15000

10000

5000
0

(e)

Fungicide Control

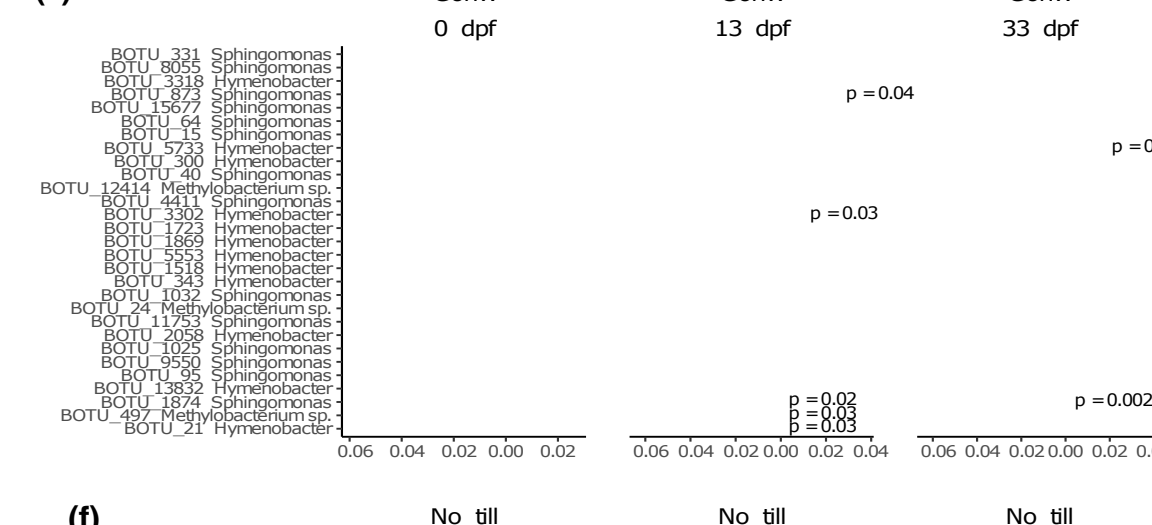

(f)

No-till

No-till

No-till
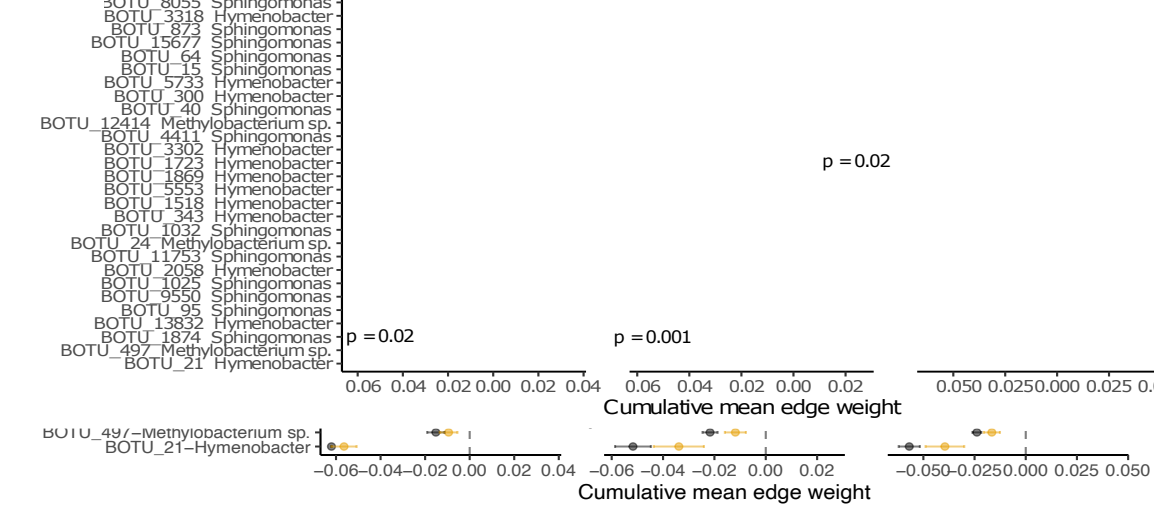


\section{Fig. 4 Indirect effects of fungicides on prokaryotic communities from altered}

1008 Bulleribacideaceae diversity on soybean leaves. Relationship of observed versus predicted

1009 Bulleribasidiaceae richness in conventional (a) or no-till (c) from random forest models using

1010 prokaryote OTU abundance in fungicide treated plots. The most important $(P<0.05)$ prokaryote

1011 OTUs for random forest model accuracy in fungicide treated conventional (b) or no-till (d). The

1012 cumulative mean edge weight calculated from each sub-network of a meta-network of

1013 Bulleribacidaceae edges between Sphinogomonas, Hymenobacter, or Methylobacterium OTUs and

1014 alterations to co-occurrence strength with and without fungicides under (e) conventional and (f) no1015 till crop management. 